\title{
An empirical model for protostellar collapse
}

\author{
A. P. Whitworth and D. Ward-Thompson \\ Department of Physics \& Astronomy, Cardiff University, PO Box 913, Cardiff, UK
}

\begin{abstract}
We propose a new analytic model for the initial conditions of protostellar collapse in relatively isolated regions of star formation. The model is non-magnetic, and is based on a Plummer-like radial density profile as its initial condition. It fits: the observed density profiles of pre-stellar cores and Class 0 protostars; recent observations in prestellar cores of roughly constant contraction velocities over a wide range of radii; and the lifetimes and accretion rates derived for Class 0 and Class I protostars. However, the model is very simple, having in effect only 2 free parameters, and so should provide a useful framework for interpreting observations of pre-stellar cores and protostars, and for calculations of radiation transport and time-dependent chemistry. As an example, we model the pre-stellar core L1544.
\end{abstract}

Subject headings: stars: formation - ISM: clouds - ISM: individual (L1544)

\section{Introduction}

Observations of the earliest stages of pre-stellar and protostellar evolution constrain the parameter space within which realistic models of low-mass $\left(\mathrm{M} \lesssim 2-3 \mathrm{M}_{\odot}\right)$ star formation can operate. The pioneering work of Myers and collaborators (e.g. Myers \& Benson 1983; Benson \& Myers 1989) and subsequent searches (e.g. Clemens \& Barvainis 1988; Bourke, Hyland \& Robinson 1995; Jessop \& Ward-Thompson 2000) have identified many dense, isolated cores in which star formation appears to have recently taken place or to be about to occur. These cores can be divided into those with protostars, as deduced from their IRAS emission, and those without - the 'starless cores' (Beichman et al 1986). Subsequent study of submillimetre continuum emission from the cold dust in starless cores has identified those which are closest to forming protostars, which are labelled pre-stellar cores (Ward-Thompson et al 1994; 1999). Pre-stellar cores are believed to represent imminent or ongoing collapse, before a central star-like object has formed. Therefore they should reflect most closely the initial conditions for collapse.

Once a central star-like object forms, the source is known as a protostar. It is classified as a Class 0 protostar as long as most of the mass is still in the infalling envelope - rather than in the central star-like object plus its accretion disc (André, Ward-Thompson \& Barsony 1993 hereafter AWB93). After half the mass of the envelope has fallen in, the source is referred to as a Class I protostar (Lada \& Wilking 1984; Lada 1987). Eventually most of the envelope has been 
accreted, or dissipated, and the source is referred to as a Class II source, which is a classical T Tauri star with a circumstellar disc. In the final pre-main-sequence stage the inner part of the disc has disappeared, and it becomes a Class III source, which is a weak-line T Tauri star (André \& Montmerle 1994). Any satisfactory model of star formation must reproduce the observed properties of these protostars, starting from the initial conditions observed in pre-stellar cores. In particular, we note the following apparently generic features:

1. Density profiles: Pre-stellar cores have flat central density profiles ranging from constant density to $\rho \propto r^{-1}$ in their innermost few thousand AU (Ward-Thompson et al. 1994; André, Ward-Thompson \& Motte 1996; Ward-Thompson, Motte \& André 1999). The density profiles then steepen towards their edges, demonstrating gradients up to $\rho \propto r^{-4}$ or even $r^{-5}$ at radii $\gtrsim 10,000$ AU (Abergel et al. 1996; Bacmann et al. 2000). A single-index power-law may fit a portion of the density profile of a given core, but no core can be fitted by a single power-law throughout.

2. Time-scales: Proceeding chronologically, the pre-stellar core phase lasts a few $10^{6}$ years. The Class 0 protostellar phase then lasts a few $10^{4}$ years, and is characterized by a very high accretion rate $\left(\gtrsim 10^{-5} M_{\odot} \mathrm{yr}^{-1}\right)$, which delivers at least half of the final stellar mass. Finally, the Class I protostellar phase lasts a few $10^{5}$ years, and is characterized by a significantly lower accretion rate $\left(\lesssim 10^{-6} M_{\odot} \mathrm{yr}^{-1}\right)$, which delivers the remainder of the final stellar mass. We note that there is a large uncertainty in these time-scales, because they are derived from statistical arguments (e.g. Beichman et al. 1986; André 1994; Ward-Thompson 1996; André, Ward-Thompson \& Barsony 2000 - hereafter AWB00) and the statistics may be influenced by selection effects.

3. Velocity fields: There are already significant inward velocities in the outer layers of the prestellar core L1544, even though there is no evidence for a central protostar having formed yet (Tafalla et al. 1998; Williams et al. 1999). Inward velocities have now also been detected in several other pre-stellar cores (e.g. Lee, Myers \& Tafalla 1999; Gregersen \& Evans 2000), and it appears that a few per cent of cores may already be contracting.

These observations cast considerable doubt on the star formation model proposed by Shu, Adams \& Lizano (1987) in which a singular isothermal sphere (SIS) undergoes inside-out collapse and delivers a constant accretion rate of order $a^{3} / G$ onto the central protostar (where $a$ is the isothermal sound speed). Additionally, there are many strong theoretical reasons (e.g. Whitworth et al. 1996) for questioning the relevance of the SIS model. In particular, there is a low likelihood that nature can assemble anything approaching a SIS in the first place, due to its innate instability (e.g. Whitworth \& Summers 1985). Also, the pre-disposition of a SIS to collapse to a single star contrasts starkly with the large fraction of newly-formed stars observed to be in binary systems (e.g. Simon et al. 1995). Therefore it is appropriate to look for alternative models. In this paper we present a simple analytic model for protostar formation satisfying the above observations. Our 
model is non-magnetic, and therefore provides a useful complement to magnetic models, such as those of Safier, McKee \& Stahler (1997) and Ciolek \& Basu (2000).

However, not all of the observations present a coherent picture. There is a well-known 'luminosity problem' for Class 0 \& I sources (e.g. Kenyon et al. 1990), namely that their low luminosities imply low accretion rates if the material is accreting directly onto the protostellar surface. Low accretion rates imply long lifetimes, which appear to be incompatible with the relatively small number of known Class 0 \& I sources. One possible explanation in the case of Class I sources is that the accretion rate remains low throughout the Class I phase, and most of the remaining protostellar envelope is dissipated in some way other than by being accreted onto the central protostar. For instance, it might be converted into lower-mass companions, as in the simulations of Boffin et al. (1998) and Watkins et al. (1998a \& b).

Alternatively, Kenyon \& Hartmann (1995) explain it by arguing that accretion takes place in a fairly uniform manner from the envelope onto a disc, and is then episodic in nature from the disc onto the central protostar. Short-lived (and therefore infrequently observed) rapid accretion episodes (manifesting themselves as FU Orionis activity) are interspersed with long periods of slow, or even negligible, accretion during which matter piles up at the inner edge of the accretion disc. Thus protostars spend the majority of their time in a 'lower luminosity' phase, only flaring up briefly when the accretion disc becomes unstable and matter is rapidly deposited onto the protostar.

This explanation was used by Kenyon \& Hartmann (1995) to account for the low luminosity of Taurus Class I sources, but it could equally well apply to the Class 0 phase. In our model we treat the central 'protostar+disc' system simply as a point source, since in most relevant $\mathrm{mm} / \mathrm{submm}$ observations the protostar and surrounding disc are unresolved, and we simply consider accretion from the envelope onto the central 'protostar+disc' system. Episodic accretion from disc onto protostar solves the 'luminosity problem' for our model as happily as for any other star formation model. In a future version of the model we plan to include the effects of angular momentum, at which point we will be able to model the disc separately from the central protostar (c.f. Cassen \& Moosman 1981; Terebey, Shu \& Cassen 1984).

The paper is laid out as follows. Section 2.1 describes the mathematical model. Section 2.2 details how the above observations constrain the model parameters. Section 3 gives a specific example of using the model to compare with one particular source, L1544: Section 3.1 lists the relevant observations of L1544; Section 3.2 illustrates the specific model; Section 3.3 compares the predictions of this model with measured lifetimes of other pre-stellar cores and protostars. Section 4 compares our model to previously published star formation models. Section 5 presents our main conclusions. 


\section{The model}

\subsection{General analysis}

The model assumes that when a pre-stellar core becomes unstable against collapse at time $t=0$, it is static and approximates to a Plummer-like density $(\rho)$ profile (Plummer 1911), in terms of radius $r$, of the form

$$
\rho(r, t=0)=\rho_{\text {flat }}\left[\frac{R_{\text {flat }}}{\left(R_{\text {flat }}^{2}+r^{2}\right)^{1 / 2}}\right]^{\eta} .
$$

Thus the initial density is approximately uniform at $\sim \rho_{\text {flat }}$ for $r \ll R_{\text {flat }}$, and it falls off as $r^{-\eta}$ for $r \gg R_{\text {flat }}$. We note parenthetically that the true Plummer Sphere, which is widely used in work on star and galaxy clusters, corresponds to the specific case $\eta=5$. It is for this reason that we describe equation 1 as 'Plummer-like'. We choose this form, not because it is predicted by detailed thermodynamic considerations or simulations of core formation, but because it seems to capture with a minimum number of free parameters - the essential observed properties of pre-stellar cores. It is mathematically simple and - as we now show - it leads to predictions which accord well with the observational constraints.

We compute the subsequent evolution of this model pre-stellar core on the assumption that it has negligible pressure, and therefore undergoes freefall collapse, starting at time $t=0$. For this purpose, it is convenient to adopt a Lagrangian formulation, i.e. to switch from $(r, t)$ to $(M, t)$ as the independent variables. Given the initial density profile $\rho(r, t=0)$ in equation (1), we can integrate to obtain the initial mass profile

$$
M(r, t=0)=\int_{r^{\prime}=0}^{r^{\prime}=r} \rho\left(r^{\prime}, t=0\right) 4 \pi r^{\prime 2} d r^{\prime} .
$$

This can then be inverted to obtain $r(M, t=0)$, i.e. the initial radius of the shell with mass $M$ interior to it. The freefall time of the shell is

$$
t_{\mathrm{FF}}(M)=\frac{\pi}{2}\left[\frac{r^{3}(M, 0)}{2 G M}\right]^{1 / 2}
$$

and at this time the shell delivers a rate of infall

$$
\dot{M}(M)=\left[\frac{d t_{\mathrm{FF}}}{d M}(M)\right]^{-1}
$$




$$
=\frac{64 G M^{2} \rho(M, 0) t_{\mathrm{FF}}(M)}{\pi\left[3 M-4 \pi r^{3}(M, 0) \rho(M, 0)\right]}
$$

onto the centre. To obtain the density profile at an arbitrary (positive) time $t$, we can use the equation for freefall from rest. For all shells having $t_{\mathrm{FF}}(M)>t$, the instantaneous radius $r(M, t)$ is given by

$$
\frac{t}{t_{\mathrm{FF}}(M)}=\frac{2}{\pi}\left\{\cos ^{-1}\left(\left[\frac{r(M, t)}{r(M, 0)}\right]^{1 / 2}\right)+\left[\frac{r(M, t)}{r(M, 0)}\right]^{1 / 2}\left[1-\frac{r(M, t)}{r(M, 0)}\right]^{1 / 2}\right\}
$$

Knowing $r(M, t)$, we can calculate the instantaneous density profile from

$$
\rho(M, t)=\left\{\left.4 \pi r^{2}(M, t) \frac{\partial r}{\partial M}\right|_{t}\right\}^{-1} .
$$

We can also compute the instantaneous velocity profile from

$$
v(M, t)=\left\{\frac{2 G M[r(M, 0)-r(M, t)]}{r(M, 0) r(M, t)}\right\},
$$

and the instantaneous column-density at impact parameter $b$ from

$$
\Sigma(b, t)=\frac{1}{2 \pi} \int_{M=M(b, t)}^{M=M_{\text {tot }}} \frac{d M}{r(M, t)\left[r^{2}(M, t)-b^{2}\right]^{1 / 2}} .
$$

The simplest computational strategy is to generate a dense table of values of $r(m, 0), \rho(M, 0), M$, $t_{\mathrm{FF}}(M), \dot{M}(M)$; and a second dense table of values of $\xi \equiv r(M, t) / r(M, 0)$ and $\tau \equiv t / t_{\mathrm{FF}}(M)$; then to use these for interpolation.

\subsection{Observational constraints}

In order to reproduce the observations described above, we must adopt $\eta \gtrsim 4$. The choice of $\eta$ is dictated by the relative durations of the Class 0 and Class I protostellar phases, and the fact that at least half the final stellar mass is delivered during the Class 0 phase (see AWB00 and references therein). If a much smaller value of $\eta$ were adopted, the accretion rate would not decrease sufficiently rapidly going from the Class 0 to the Class I protostellar phase, i.e. by about an order of magnitude between the infall of the first half of the envelope mass (the Class 0 phase) and the infall or dissipation of the rest (the Class I phase). Indeed, for $\eta \leq 3$, the mass of the protostellar core diverges, and the accretion rate falls off less rapidly than $\mathrm{t}^{-1}$. 
If a much larger value of $\eta$ were adopted, the accretion rate would fall off very rapidly, but this would only happen after almost all the envelope mass had fallen in; in other words, the accretion rate would switch rather abruptly from being substantial to being negligible. This would only be acceptable if stars assembled most of their mass rapidly during the Class 0 phase and right at the beginning of the Class I phase, with the rest of the Class I phase contributing very little to the final mass. This may not be completely ruled out by the observations, particularly if the outer envelopes of protostars are dispersed rather than being accreted, but it would be difficult to reconcile with the luminosities of Class I protostars. Hence, we conclude that the optimum value of $\eta$ is 4 , and we fix it at this value; therefore it is no longer a free parameter of the model.

The value of $\rho_{\text {flat }}$ (the central density at the onset of freefall collapse) is dictated not only by observations of pre-stellar cores, but also by the duration of the Class 0 phase of a few $10^{4}$ years. With $\eta=4$, the duration of the rapid accretion phase (i.e. the Class 0 phase) is comparable to the initial freefall time at the centre of the pre-stellar core, $t_{\mathrm{o}} \equiv t_{F F}(M=0)$, so we require

$$
t_{\mathrm{o}}=\left[\frac{3 \pi}{32 G \rho_{\text {flat }}}\right]^{1 / 2} \sim \text { few } 10^{4} \text { years },
$$

which gives $\rho_{\text {flat }} \sim 3 \times 10^{-18} \rightarrow 3 \times 10^{-17} \mathrm{~g} \mathrm{~cm}^{-3}$. Finally, $R_{\text {flat }}$ is fixed by the total mass of the pre-stellar core being modelled.

\section{Detailed results and comparison with L1544}

\subsection{L1544 observations}

For the purposes of illustration, we have chosen to model the evolution of the pre-stellar core L1544, because it is one of the most carefully observed - and hence one of the most tightly constrained - pre-stellar cores. Its velocity field has been studied in detail (Tafalla et al. 1998; Williams et al. 1999). Its density profile and total mass $\left(M_{\text {tot }} \sim 8 M_{\odot}\right)$ have been deduced from millimetre continuum observations (Ward-Thompson et al. 1999). And its far-infrared emission (as observed by the Infrared Space Observatory, ISO) shows it to be roughly isothermal (AWB00). We note that since our model is driven purely by self-gravity, it is very simple to re-scale the results to fit other sources, by altering $\rho_{\text {flat }}$ and $R_{\text {flat }}$. The masses then scale as $M \propto \rho_{\text {flat }} R_{\text {flat }}^{3}$, time-scales as $t \propto \rho_{\text {flat }}^{-1 / 2}$, velocities as $v \propto \rho_{\text {flat }}^{1 / 2} R_{\text {flat }}$, and accretion rates as $\dot{M} \propto \rho_{\text {flat }}^{3 / 2} R_{\text {flat }}^{3}$.

Tafalla et al. (1998) infer that the central core in L1544 is pre-stellar but probably close to forming a Class 0 protostar. From a detailed analysis of the profiles of various molecular lines in particular CS $(J=2 \rightarrow 1)$, which is self-absorbed, and $\mathrm{C}^{34} \mathrm{~S}(J=2 \rightarrow 1)$, which is not - they argue that the outer layers of L1544 are falling inwards. Specifically, they deduce that gas at radii $r \sim 1-4 \times 10^{4} \mathrm{AU}$ is moving inwards at speed $v \sim 0.08 \mathrm{~km} \mathrm{~s}^{-1}$. Williams et al. (1999) detect similar infall speeds in their interferometric observations of $\mathrm{N}_{2} \mathrm{H}^{+}(\mathrm{J}=1 \rightarrow 0)$, and infer gas infalling 
at $\sim 0.08 \mathrm{~km} \mathrm{~s}^{-1}$ on much smaller scales of $\sim 2,000$ AU. We shall refer to the region 2,000 AU $\lesssim r \lesssim 40,000 \mathrm{AU}$ as 'the inflow region'.

Based on these observations, Williams et al. (1999) claimed that L1544 is inconsistent with ambipolar diffusion models. Ciolek \& Basu (2000) then presented a model that they claimed could explain the observations, by taking initial conditions that are close to magnetically critical. SCUBA polarimetry observations of L1544 (Ward-Thompson et al. 2000) showed a magnetic field that does not align with the short axis of the core. This can also be explained by initial conditions close to magnetically critical. However, Bacmann et al. (2000) presented ISOCAM data that show that L1544 has a steep radial density profile in its outer edges. This requires highly magnetically sub-critical initial conditions to explain it. Clearly there is a contradiction here. One possible explanation is that the magnetic field may not be playing a significant role, and we are simply seeing gravitational contraction prior to the formation of a protostar. Thus we shall attempt to model the above mentioned data of L1544, without invoking a magnetic field, using $\eta=4$, $\rho_{\text {flat }}=3 \times 10^{-18} \mathrm{~g} \mathrm{~cm}^{-3}$ and $R_{\text {flat }}=5350 \mathrm{AU}$ ( $\equiv 0.026 \mathrm{pc}$ ), giving a total core mass of $M_{\text {tot }}=8 M_{\odot}$. We stress that these values are chosen to match this one particular core. Our presumption is that other cores have a range of values of $\rho_{\text {flat }}$ and $R_{\text {flat }}$.

\subsection{Model predictions for L1544}

Figure 1 shows the evolution of the volume density profile, through the end of the pre-stellar core phase and the start of the Class 0 phase, as a log-log plot of number density $\left(\right.$ in $\mathrm{H}_{2}$ per $\mathrm{cm}^{3}$ ) versus radius (in $\mathrm{AU}$ ). The profiles at different epochs are labelled according to the amount of time which has elapsed as a fraction of the initial free-fall time at the centre of the pre-stellar core, $t_{\mathrm{o}}$. Hence $\left(t / t_{\mathrm{o}}\right)=0$ represents the initial conditions; and $\left(t / t_{\mathrm{o}}\right)=1$ represents the profile after one central free-fall time, at the birth of the central point-mass - i.e. the end of the pre-stellar phase and the start of the Class 0 phase.

The dashed line shows the measured radial density profile of L1544 deduced from millimetre continuum observations (Ward-Thompson et al. 1999). It can be seen from Figure 1 that the model density profile starts relatively flat in the centre, and steepens towards the edge, in approximate agreement with the observed radial density profile. The crosses mark the density and radius at the positions of the detected inflow, as deduced by Tafalla et al. (1998) and Williams et al. (1999). These are also consistent with the model predictions at early times.

As the model evolves, it can be seen that the central density slowly increases. As one freefall time is approached the central density profile starts to steepen, asymptotically approaching $\rho \propto r^{-3 / 2}$. The outermost profile stays virtually unchanged at its initial $\rho \propto r^{-4}$. After the formation of a central point-mass (at one free-fall time), the magnitude of the density decreases slowly as more of the circumstellar material accretes onto the central point-mass, but the shape

of the inner density profile stays similar at $\rho \propto r^{-3 / 2}$. This is consistent with the recent results 
of Chandler \& Richer (2000), who conclude on the basis of SCUBA images that young Class 0 protostars approximate to $\rho \propto r^{-3 / 2}$ on scales of $300-10,000 \mathrm{AU}$.

Figure 2 shows the evolution of the inward radial velocity profile, through the pre-stellar stage and the start of the Class 0 phase, as a log-log plot of velocity $-v$ (in $\mathrm{km} \mathrm{s}^{-1}$ ) versus radius (in $\mathrm{AU}$ ). Once again, the profiles at different epochs are labelled according to the amount of time which has elapsed as a fraction of the initial free-fall time at the centre of the pre-stellar core, $t_{\mathrm{o}}$. The initial conditions are static, so the velocity is zero everywhere at $\mathrm{t}=0$. Between times of roughly $\left(t / t_{\mathrm{o}}\right)=0.3$ to $\left(t / t_{\mathrm{o}}\right)=0.7$ (the middle of this range is shown), the central velocity profile approximates to $v \propto r$, and the outer velocity profile approximates to $v \propto r^{-2}$. Throughout this phase the absolute value of velocity increases monotonically with time at all radii.

After the formation of a central protostar at one free-fall time the central velocity profile tends towards $v \propto r^{-1 / 2}$. However, the outer velocity profile remains relatively unchanged in form, although it carries on increasing in magnitude. The crosses on Figure 2 represent the velocity and radius at the two positions in the inflow region deduced by Tafalla et al. (1998) and Williams et al. (1999) from their respective observations. They are consistent with the model predictions at $\left(t / t_{\mathrm{o}}\right) \simeq 0.5$.

Figure 3 shows how the accretion rate varies with time measured from the onset of freefall collapse, now plotted on a linear scale of $\dot{M}$ versus $t$. We note that the shape of this curve is determined solely by the choice of $\eta$. The choices of $\rho_{\text {flat }}$ and $R_{\text {flat }}$ only influence the scaling. For any flat-topped initial density profile, there is a pause between the onset of freefall collapse at $t=0$, and the onset of accretion onto a central point-mass at $t=t_{\mathrm{o}}$ (remember that we are treating the central protostar and its circumstellar disc merely as a point source). At this point the contracting pre-stellar core phase ends, the accretion rate becomes non-zero and the object becomes a Class 0 source.

Hence this detailed comparison, using the specific example of L1544, shows remarkably good agreement between our model and all of the measured quantities. The apparently roughly uniform velocity profile is well reproduced by a collapsing Plummer sphere at $t / t_{\mathrm{o}} \simeq 0.5$. The measured density profile, both from the continuum and the spectroscopy, is also consistent with $t / t_{\mathrm{o}} \simeq 0.5$. Thus the pre-stellar core L1544 is consistent with our model, in the earliest stages of collapse.

\subsection{Comparison of lifetimes}

We can compare the various lifetimes predicted by the model illustrated in Figure 3 with those derived observationally from statistical arguments (although we note that such arguments carry large uncertainties). We have defined the time $t=0$ to be the point at which a pre-stellar core begins to contract. We will refer to the time from $t=0$ until $t=t_{\mathrm{o}}$ as the 'contracting prestellar core phase'. Note that this is not the same as the total pre-stellar core phase, because most pre-stellar cores show no evidence of infall motions and therefore are presumably not contracting. 
Our model says nothing about the total pre-stellar core lifetime, merely about the lifetime of the contracting pre-stellar core phase. Ward-Thompson et al. (1994) estimated the lifetime of prestellar cores detected in the submm continuum to be $\sim 10^{6}$ years. This was based on a total starless core lifetime of a few $10^{6}$ years, derived from the survey of Beichman et al. (1986).

Lee, Myers \& Tafalla (1999) surveyed a total of 220 starless and pre-stellar cores and found definite evidence for collapse in only 7 of those. There was tentative evidence for contraction in a further 10 cores, but there was also some doubt about the exact frequencies of some of the transitions used in that survey, calling into question some of the tentative detections, so we do not consider them here. If the fraction of contracting pre-stellar cores is 7 out of 220 , as suggested by Lee et al. (1999), then the contracting pre-stellar core phase lasts for only $3 \%$ of the total starless and pre-stellar core phase. Hence the lifetime of the contracting pre-stellar core phase is $\sim 3-10 \times$ $10^{4}$ years. It can be seen from Figure 3 that our model predicts a value for this phase consistent with the lower end of this range.

Gregersen \& Evans (2000) observed a subset of sources from the list of Ward-Thompson et al. (1994), which in turn was drawn from the catalogue of starless cores listed by Beichman et al. (1986), and discovered six more cores with line asymmetries indicative of collapse. The list of Beichman et al. (1986) contained almost 50 starless cores, hence the result of Gregersen \& Evans (2000) appears to be implying a lifetime for the contracting pre-stellar core phase of $\sim 10 \%$ of that of the total starless core phase, or a few $10^{5}$ years. This is longer than our model predicts.

However, the fact that two different statistical studies can produce results for this timescale that differ by an order of magnitude illustrates the uncertainties inherent in such calculations. For example, the Gregersen \& Evans (2000) sample contains a great deal of biassing and selection effects, since they consciously chose sources which were already believed to be closest to forming Class 0 protostars. Hence an order of magnitude uncertainty in a statistical lifetime derived from such a sample is not surprising. Nonetheless, if a significantly larger number of contracting pre-stellar cores is discovered with improving detection techniques, and the total pre-stellar core life-time remains unchanged, then our model would have to be modified to increase the contracting pre-stellar core lifetime (see section 4 below).

After the pre-stellar core stage a central point-mass forms, and the accretion rate increases very rapidly to its maximum value, before decreasing on a time scale $\sim t_{\mathrm{o}}$. We have marked on Figure 3 the points at which various fractions of $M_{\text {tot }}$ have been accreted. Remember that the Class $0 /$ I borderline occurs at $M / M_{\text {tot }}=50 \%$. It can be seen that the Class 0 phase lasts for $\sim 8 \times 10^{4}$ years, and has much more rapid accretion than the Class I phase.

We can compare the Class 0 lifetime predicted by our model with the lifetimes of various sources that have been estimated from observations. AWB93 defined the Class 0/I borderline as the point where a source has accreted half of its final main-sequence mass. They used the ratio between the submillimetre luminosity $\mathrm{L}_{\text {submm }}$ and the bolometric luminosity $\mathrm{L}_{b o l}$ as an observational indicator of this, where Class 0 sources have $\mathrm{L}_{s u b m m} / \mathrm{L}_{b o l}>0.005$, and Class I sources have $\mathrm{L}_{s u b m m} / \mathrm{L}_{b o l}<$ 
0.005 .

VLA1623 has $\mathrm{L}_{\text {submm }} / \mathrm{L}_{\text {bol }} \sim 0.1$, and hence is a Class 0 object. It also has an estimated age of a few $10^{4}$ years (AWB93). We can see from Figure 3 that our model predicts that VLA1623 should have accumulated less than $50 \%$ of its mass at this at this age, consistent with its Class 0 classification. $\mathrm{L} 1527$ has a ratio of $\mathrm{L}_{\text {submm }} / \mathrm{L}_{\text {bol }}$ of 0.007 , making it a borderline Class 0/I object. It has an estimated age of $\sim 10^{5}$ years (Ohashi et al. 1997). From Figure 3 we can see that at this age, our model would predict that L1527 should have accumulated about $50 \%$ of its mass, consistent with its borderline Class $0 /$ I categorization.

Thus we see that the model can reproduce easily the density profile and dynamics of the prestellar core L1544, as well as reproducing the statistical lifetimes of contracting pre-stellar cores and Class 0 \& I protostars, and the rapid growth of a protostar during the Class 0 phase. We note once again that the model illustrated in Figures 1-3 is merely one example of the generic class of Plummer-like models that we are presenting here. Varying the central density $\rho_{\text {flat }}$ and the radius $R_{\text {flat }}$ produces a family of solutions, and different solutions may be appropriate for different star-forming regions.

\section{Model tests and comparison with other models}

The model we have developed here is simple, in the sense that it has effectively only 2 free parameters $\left(\rho_{\text {flat }}\right.$ and $R_{\text {flat }}$ ). It represents well the observed density profiles of pre-stellar cores and Class 0 protostars. It reproduces the velocity field deduced for the pre-stellar core L1544, and it matches the accretion rates and lifetimes inferred statistically for Class 0 and Class I protostars. It may therefore provide a useful framework for interpreting observations of pre-stellar cores and protostars, and for calculations of radiation transport and time-dependent chemistry.

Discriminating tests of the model will require further observations, such as larger surveys to improve the statistics upon which the time-scales and accretion rates are based, and more sensitive probes of the density and velocity profiles which must be reproduced. If such subsequent surveys continue to demonstrate what the surveys thus far have indicated - namely that the relative lifetimes of Class 0 \& I protostars are as we have adopted here, and that the accretion rate decreases with time - then this model will continue to prove useful. If, however, more sensitive observations of pre-stellar cores show them to have density and velocity profiles which (after modelling their collapse) cannot reproduce the inferred protostellar life-times, then this would refute the model.

Our model is somewhat similar to that developed by Henriksen, André \& Bontemps (1997), except that we do not take the density to be exactly uniform in the central region, and so we avoid an infinite accretion rate during the Class 0 phase. This has three advantages: first, it reduces the number of free parameters (our $\eta$ replaces their $D_{1}, D_{2}$ and $r_{\mathrm{b}} / r_{\mathrm{N}}$ ); second, it allows us to fit the observationally inferred density and velocity profiles of pre-stellar cores; and third, it enables us to use estimates of the duration of the Class 0 phase to constrain the central density at the onset of 
collapse.

We note that the models of cores in thermal and mechanical equilibrium derived by Falgarone \& Puget (1985) and by Chièze \& Pineau des Forêts (1987) have structures very similar to what we are proposing, namely a flat density profile in the centre, and a steep fall-off further out with $-d \ell n[\rho] / d \ell n[r] \simeq 4$. Similar density profiles are also predicted by ambipolar diffusion models (e.g. Ciolek \& Mouschovias 1994; Safier, McKee \& Stahler 1997), but these models have difficulty reproducing both the relatively short contracting pre-stellar lifetimes and the relatively large inflow velocities.

Finally, we emphasize that, unless contraction is driven by external pressure (e.g. Myers \& Lazarian 1998), the freefall collapse of an initially flat-topped density configuration is almost certainly the least extreme way of delivering the rapid accretion rates inferred for Class 0 protostars. Any refinements to the model, such as the inclusion of thermal pressure, turbulence, a magnetic

field, or rotation, are likely to slow the collapse down, in which case the pre-stellar core would have to start collapsing from a denser initial state.

\section{Conclusions}

In this paper we have proposed a new analytic model for the initial conditions of protostellar collapse in moderately isolated regions of star formation. The model is non-magnetic, and is based on a Plummer-like radial density profile as its initial condition. The model fits:

1. the observed density profiles of pre-stellar cores;

2. the observed density profiles of Class 0 protostars;

3. recent observations of roughly constant contraction velocities over a wide range of radii in the L1544 pre-stellar core;

4. the lifetimes of contracting pre-stellar cores;

5. the relative lifetimes derived for Class 0 and Class I protostars;

6. the relative inferred accretion rates of Class 0 and Class I protostars.

Moreover, the model is very simple, having in effect only 2 free parameters, $\rho_{\text {flat }}$ and $R_{\text {flat }}$ ( $\eta$ is so tightly constrained by observations that it should not be considered 'free'). Therefore it should provide a useful framework for interpreting observations of pre-stellar cores and protostars, and for calculations of radiation transport and time-dependent chemistry. We shall consider models involving external compression, rotation and magnetic fields in subsequent papers. We also plan to 
add radiative transfer to our model to allow us to model line profiles directly. We would be happy to supply copies of the model code upon request. ${ }^{1}$

\section{REFERENCES}

Abergel A., et al., 1996, A\&A 315, L329

André P., 1994, in: The Cold Universe, p. 179, eds. Montmerle Th., Lada C. J., Mirabel I. F., Trân Thanh Vân J., Editions Frontieres, Paris

André P., Montmerle T., 1994, ApJ, 420, 837

André P., Ward-Thompson D., Barsony M., 1993, ApJ, 406, 122 - AWB93

André P., Ward-Thompson D., Barsony M., 2000, in: Protostars \& Planets IV, in press, University of Arizona Press - AWB00

André P., Ward-Thompson D., Motte F., 1996, A\&A, 314, 625

Bacmann A., André P., Puget J-L., Abergel A., Bontemps S., Ward-Thompson D., 2000, A\&A, in press

Beichman C. A., Myers P. C., Emerson J. P., Harris S., Mathieu R., Benson P. J., Jennings R. E., 1986, ApJ, 307337

Benson P. J., Myers P. C., 1989, ApJS, 71, 89

Boffin H. M. J., Watkins S. J., Bhattal A. S., Francis N., Whitworth A. P, 1998, MNRAS, 300, 1189

Bontemps S., André P., Terebey S., Cabrit S., 1996, A\&A, 311, 858

Bourke T. L., Hyland A. R., Robinson G., 1995, MNRAS, 276, 1052

Cassen P., Moosman A., 1981, Icarus, 48, 353

Chandler C. J., Richer J. S., 2000, ApJ, 530, 851

Chièze J-P., Pineau des Forêts G., 1987, A\&A, 183, 98

Ciolek G. E., Mouschovias T. Ch., 1994, ApJ, 425, 142

Ciolek G. E., Basu S., 2000, ApJ, 529, 925

Clemens D. P., Barvainis R., 1988, ApJS, 68, 257

\footnotetext{
${ }^{1}$ For a copy of the model code, email: ant@astro.cf.ac.uk
} 
Falgarone E., Puget J-L., 1985, A\&A, 142, 157

Gregersen E. M., Evans N. J., 2000, ApJ, in press

Henriksen R., André P., Bontemps S., 1997, A\&A, 323, 549

Kenyon S. J., Hartmann L. W., 1995, ApJSS, 101, 117

Kenyon S. J., Hartmann L. W., Strom K. M., Strom S.E., 1990, AJ, 99, 869

Jessop N. E., Ward-Thompson D., 2000, MNRAS, 311, 63

Lada C. J., 1987, in: IAU Syposium 115, Star Forming Regions, p. 1, eds: Peimbert M., Jugaku J., Reidel, Dordrecht

Lada C. J., Wilking B., 1984, ApJ, 287, 610

Lee C. W., Myers P. C., Tafalla M., 1999, ApJ, 526, 788

Myers P. C., Benson P. J., 1983, ApJ, 266, 309

Myers P. C., Lazarian A., 1998, ApJ, 507, L157

Ohashi N., Hayashi M., Ho P. T. P., Momose M., 1997, ApJ, 475, 211

Plummer H. C., 1911, MNRAS, 71, 460

Safier P. N., McKee C. F., Stahler S. W., 1997, ApJ, 485, 660

Shu F. H., Adams F. C., Lizano S., 1987, ARA\&A, 25, 23

Simon M., Ghez A. M., Leinert Ch., Cassar, L., Chen W. P., Howell R. R., Jameson R. F., Matthews K., Neugebauer G., Richichi, A., 1995, ApJ, 443, 625

Tafalla M., Mardones D., Myers P. C., Caselli P., Bachiller R., Benson P. J., 1998, ApJ, 504, 900

Terebey S., Shu F. H., Cassen P., 1984, ApJ, 286, 529

Ward-Thompson D., 1996, Ap\&SS, 239, 151

Ward-Thompson D., Kirk J. M., Crutcher R. M., Greaves J. S., Holland W. S., André P., 2000, ApJ, submitted

Ward-Thompson D., Motte F., André P., 1999, MNRAS 305, 143

Ward-Thompson D., Scott P. F., Hills R. E., André P., 1994, MNRAS, 268, 276

Watkins S. J., Bhattal A. S., Boffin H. M. J., Francis N., Whitworth A. P., 1998a, MNRAS, 300, 1205 
Watkins S. J., Bhattal A. S., Boffin H. M. J., Francis N., Whitworth A. P., 1998b, MNRAS, 300, 1214

Whitworth A. P., Bhattal A. S., Francis N., Watkins S. J., 1996, MNRAS, 283, 1061

Whitworth A. P., Summers D., 1985, MNRAS 214, 1

Williams J. P., Myers P. C., Wilner D. J., Di Francesco J., 1999, ApJ, 513, L61 
Fig. 1.- Volume-density profiles. Each curve is marked with the value of $t / t_{\mathrm{o}}$, where the central point-mass forms at $t=t_{\mathrm{o}}$, so $t / t_{\mathrm{o}}<1$ corresponds to contracting pre-stellar cores, and $t / t_{\mathrm{o}}>1$ corresponds to young Class 0 protostars. The dashed line shows the density profile of L1544 derived from IRAM $1.3 \mathrm{~mm}$ continuum mapping by Ward-Thompson et al. (1999). The crosses are the volume-density and radius values inferred by Tafalla et al. (1998) and Williams et al. (1999) for L1544. Hence all of these observations of L1544 are consistent with $t / t_{\mathrm{o}} \sim 0.5$.

Fig. 2. - Inward radial velocity profiles. Each curve is marked with the value of $t / t_{\mathrm{o}}$, as in Figure 1. The crosses represent the infall velocities inferred by Tafalla et al. (1998) and Williams et al. (1999) for L1544. Once again a value of $t / t_{\mathrm{o}} \sim 0.5$ is consistent with the observations.

Fig. 3.- The accretion rate, in units of $10^{-5} M_{\odot} \mathrm{yr}^{-1}$, plotted against time, in units of $10^{4}$ yrs. The borderline between pre-stellar cores and Class 0 protostars occurs when the accretion rate first becomes non-negligible. The tick marks indicate the fraction of the total mass which has reached the central protostar. The Class 0/I borderline occurs when this fraction is $50 \%$. 


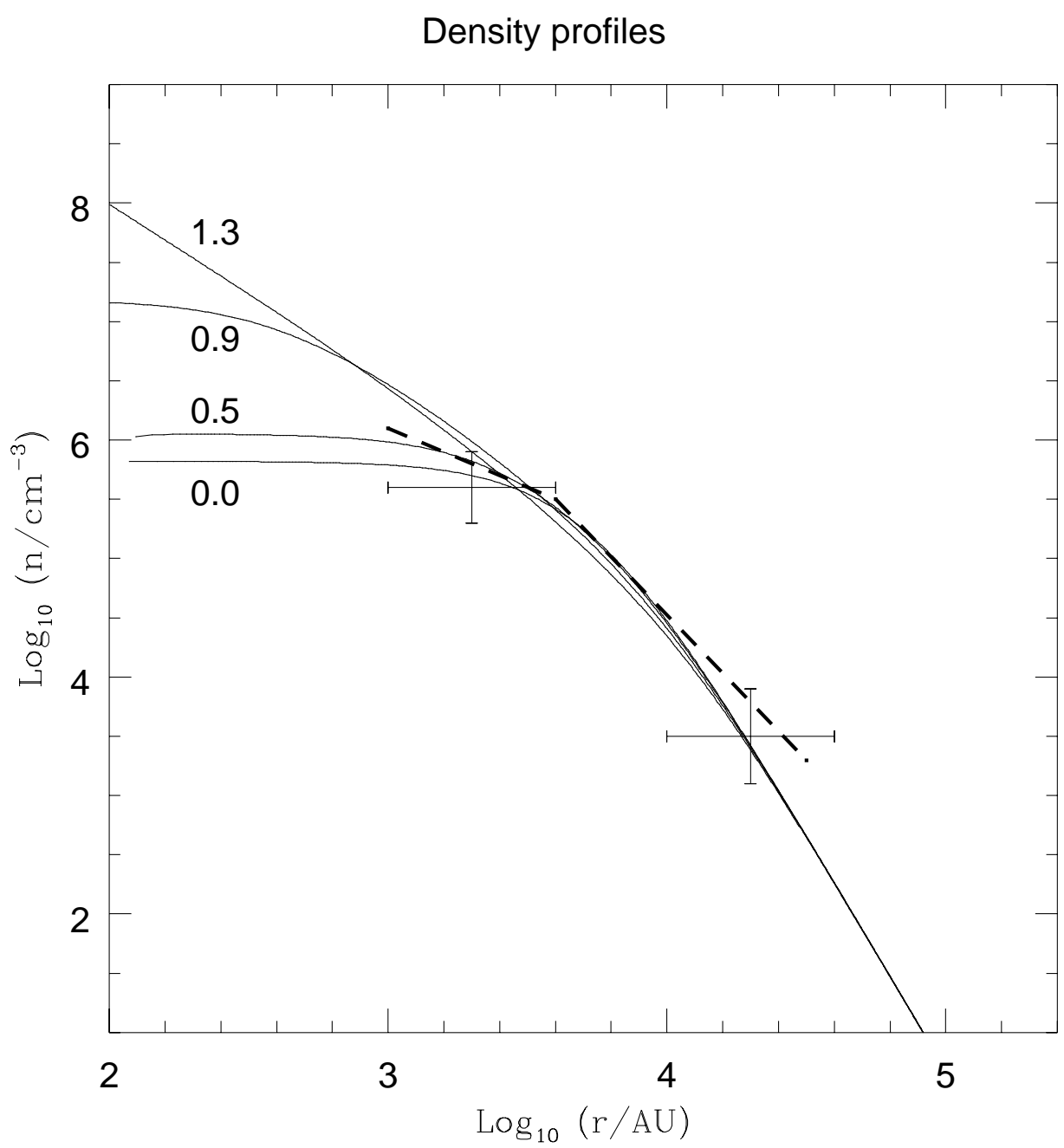




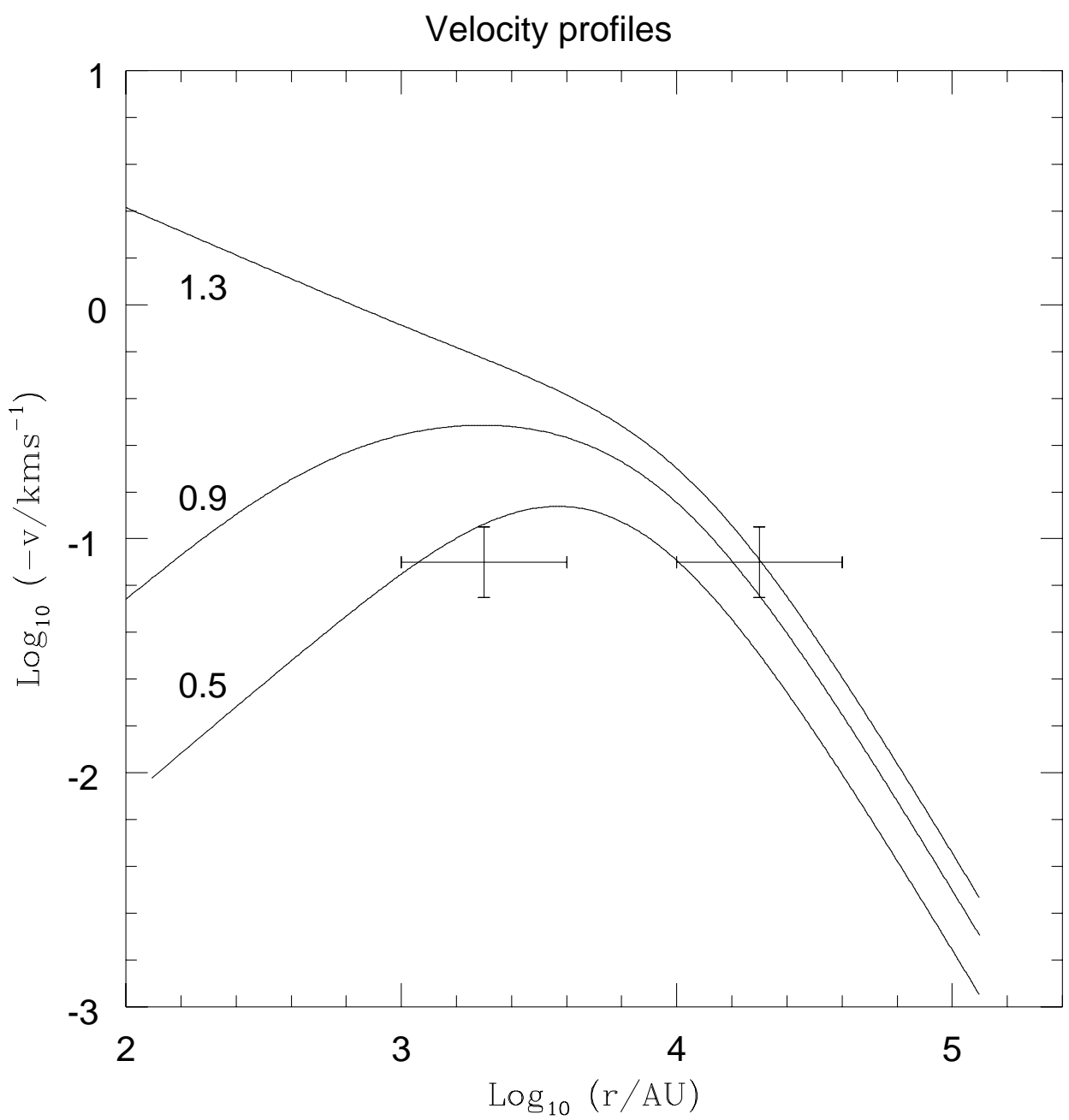




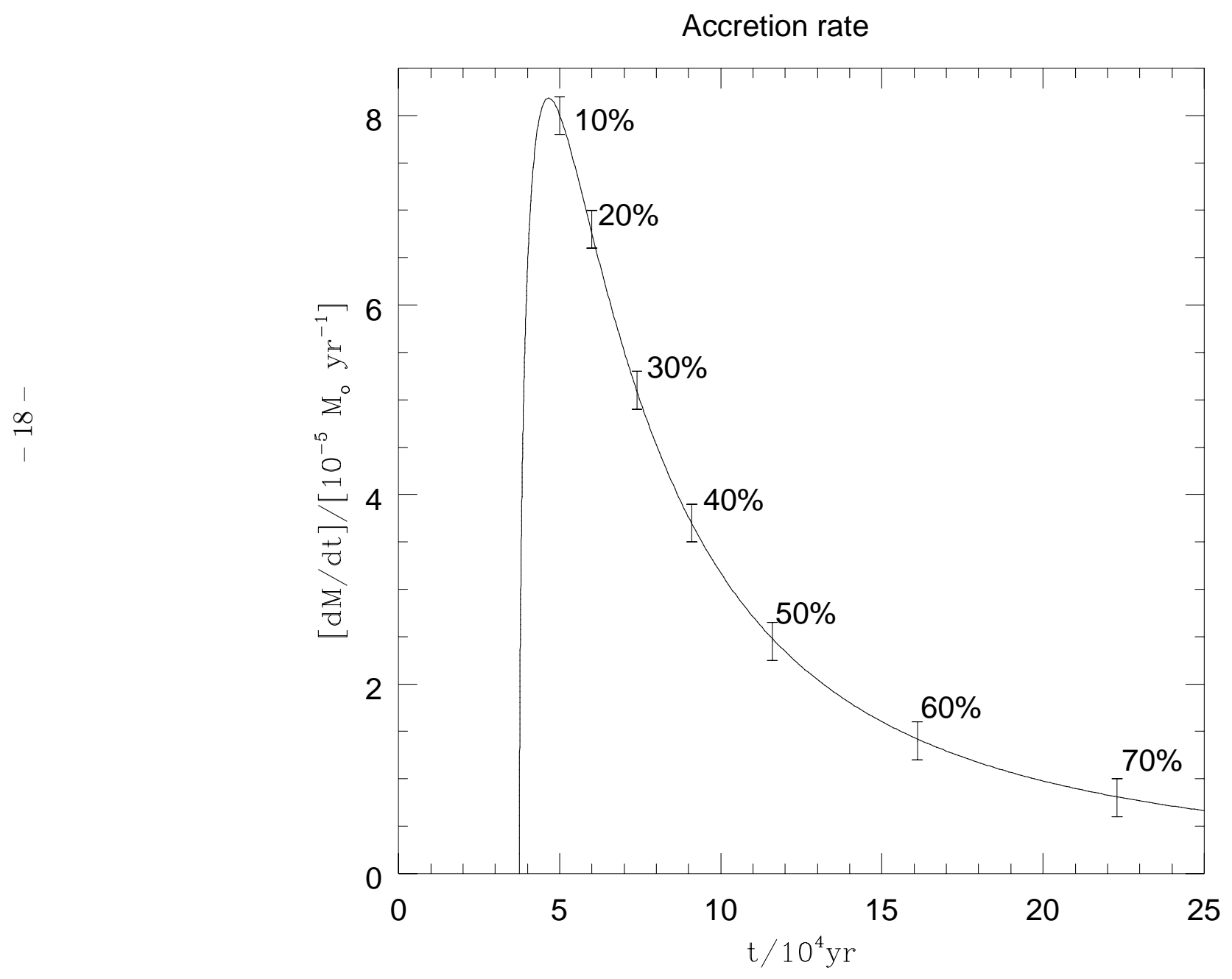

